\title{
Improved mapping strategy to better inform policy on the control of schistosomiasis and soil-transmitted helminthiasis in Sierra Leone
}

Mary Hodges ${ }^{1 *}$, Nsa Dada ${ }^{2}$, Anna Wamsley ${ }^{2}$, Jusufu Paye $^{3}$, Emanuel Nyorkor ${ }^{4}$, Mustapha Sonnie $^{1}$, Guy Barnish $^{2}$, Moses Bockarie ${ }^{2}$ and Yaobi Zhang ${ }^{5}$

\begin{abstract}
Background: Schistosomiasis and soil-transmitted helminthiasis (STH) are endemic in Sierra Leone confirmed by national mapping in 2008. To better inform planning of preventive chemotherapy strategy, another survey was conducted before mass drug administration (MDA) in seven districts according to the mapping results or local knowledge. Fifty-nine chiefdoms and one school in every chiefdom were selected. Thirty school children aged 9-14 years from each school (total: 1760) were examined by parasitological methods for infection with Schistosoma mansoni and STHS.
\end{abstract}

Results: The overall prevalence of S. mansoni was 40.2\% (95\% confidence interval (Cl): 37.9-42.5\%), particularly in Kailahun (63.3\%), Kenema (46.7\%), Koinadugu (41.9\%) and Kono (71.7\%). The results demonstrated the focal distribution of S. mansoni in Bo, Tonkolili and Bombali districts with prevalence ranging from 0.0-63.3\%, 3.3-90.0\% and 0.0-67.9\% respectively. The arithmetic mean intensity of S. mansoni infection was 95.4 epg (95\% Cl: 61.4-129.5 epg), Heavy mean intensity of infection was found in Kailahun (120.2 epg), Kenema (104.5 epg), Koinadugu (112.3 epg) and Kono (250.3 epg). Heavy or moderate infection with S. mansoni occurred in 20.7\% of children examined. Hookworm prevalence was moderate: 31.2\% (95\% Cl: 29.1-33.4\%), but high in Bo (50.0\%) and Tonkolili (56.7\%). Hookworm intensity of infection was light with a mean epg of 53.0 (95\% Cl: 38.4-67.7 epg). Prevalence and intensity of Ascaris lumbricoides (1.5\%, $17.8 \mathrm{epg}$ ) and Trichuris trichiura (2.5\%, $20.3 \mathrm{epg}$ ) was low.

Conclusions: The prediction by previous spatial analysis that S. mansoni was highly endemic across north-eastern Sierra Leone was confirmed with a significant proportion of children heavily or moderately infected. The distribution of S. mansoni in Bo, Tonkolili and Bombali districts ranged widely, highlighting the importance of considering the nature of focal transmission in national mapping exercises. These results were used to refine the MDA for schistosomiasis control to chiefdom implementation units rather than the entire district in these 3 districts. The survey demonstrated that sufficient number of survey sites for schistosomiasis mapping in each district should be used to provide a better national planning of MDA activities, and that it is affordable with the contributions from all parties involved and national resources mobilized.

\section{Background}

Schistosomiasis and soil-transmitted helminthiasis (STH) inflict a significant health and socioeconomic burden on the poorest populations in rural and poor urban settings in the tropics and subtropics, in particular, in sub-Saharan Africa [1-3]. It is estimated that over

\footnotetext{
* Correspondence: mhodges@hki.org

'Helen Keller International, Freetown, Sierra Leone

Full list of author information is available at the end of the article
}

200 million people are infected with schistosomes worldwide and about two billion with STHs [2,3]. Schistosomiasis, caused by Schistosoma mansoni or S. haematobium, and STH, of particular worldwide importance, caused by Ascaris lumbricoides, Trichuris trichiura, and hookworms (Ancylostoma duodenale and Necator americanus), cause significant morbidity worldwide with a combined 43.5 million or more disability adjusted life years (DALYs) lost each year, more than those lost to malaria (36 million) and approaching those lost to

\section{() Biomed Central}


tuberculosis (47 million) [4-6]. Schistosomiasis alone could be responsible for 200000 deaths per year in subSaharan Africa, and STH could be responsible for 135 000 deaths per year globally [7].

School-aged children, young adolescents and preschool children tend to harbour the greatest number of worms, and as a result experience stunting, wasting, diminished physical fitness as well as impaired memory and cognitive abilities $[5,8]$. These adverse health consequences combine to impair childhood educational performance and reduce school attendance, leading to a reduction in future productivity and wage-earning capacity $[5,9]$. These infections also have direct and indirect effects on malaria and HIV/AIDS in developing countries where they are co-endemic [10].

Control of schistosomiasis and STH is now part of the global effort in the integrated control of the neglected tropical diseases (NTDs) which currently targets five major 'tool-ready' diseases (lymphatic filariasis, onchocerciasis, schistosomiasis, STH and trachoma) through preventive chemotherapy [11]. The drugs needed for these five NTDs are robust, safe, low-cost and available by donation from the pharmaceutical companies or by purchasing at relatively low costs. The funds are provided by a number of governmental and non-governmental organisations, notably, the United States Agency for International Development, British Government Department for International Development, and the Bill \& Melinda Gates Foundation. This drug-based intervention delivers available drugs either alone or in combination to prevent morbidity caused by these NTDs, or in some cases to eliminate the diseases. There is currently a major drive from the World Health Organization (WHO) to prepare the countries in sub-Saharan Africa for such national NTD control programmes. Geographical mapping of these NTDs is required as an essential tool for directing national intervention programmes.

Both intestinal and urinary forms of schistosomiasis are known to be endemic in Sierra Leone [12-16], as well as STHs [17-21]. Various prevalence data on STHs in school aged children are available from the 1990s, with A. lumbricoides 32-93\%, hookworm 25-43\%, and $T$. trichiura 39-75\% [22-25]. National mapping of the prevalence and distribution of intestinal schistosomiasis and STH was conducted in Sierra Leone in 2008 [26]. In the 2008 mapping survey, four survey sites (schools) per district were selected according to administrative districts using a two-staged random sampling method to avoid two schools being selected from the same chiefdom to ensure a relatively even geographical coverage throughout the country as described previously [26]. Spatial analysis predicted that $S$. mansoni was highly prevalent in Sierra Leone with a large cluster of high risk of $S$. mansoni infection (prevalence $>70 \%$ ) in the northeast half of the country where annual preventive chemotherapy (PCT) intervention was required and that hookworm infection was high across the country with a large cluster of high infection risk (prevalence $>70 \%$ ) in the north-eastern part [26]. However, it was noted that the relatively small number of randomly sampled sites (only four per district) used in the mapping might have underestimated the endemicity, particularly of schistosomiasis in the Bo and possibly Bombali districts. Therefore, this complementary survey was conducted in 2009 in seven districts before the mass drug administration (MDA) was implemented.

The objective of the current survey was (1) to validate the predicted prevalence maps of S. mansoni and STHs in districts where the mapping suggested that widespread MDA was needed and in particular in Bo district where local knowledge suggested that schistosomiasis was indeed endemic, (2) to refine the MDA strategy in these districts in the integrated national NTD control programme, and (3) to provide intensity of infection data for S. mansoni and STHs among school aged children in the endemic areas, which was not reported earlier.

\section{Methods}

\section{Sampling and data collection}

The survey was carried out in 2009 in seven rural districts, Bo, Bombali, Kenema, Koinadugu, Kailahun, Kono and Tonkolili. These districts were shown to be highly endemic across the districts with $S$. mansoni according to the 2008 survey and spatial prediction, except for Bo where local knowledge suggests that it is also endemic for schistosomiasis but the 2008 mapping had failed to identify this. Fifty-nine (59) chiefdoms in these seven districts $(9$ chiefdoms per district from Bo, Bombali, Kailahun and Kenema, 8 chiefdoms per district from Kono and Tonkolili, and 7 chiefdoms from Koinadugu) which were not sampled in the mapping in 2008 but were predicted to be highly prevalent with $S$. mansoni were selected. One primary school per chiefdom was sampled based on accessibility. Within each school, around 30 children aged 9-14 years who volunteered to participate were enrolled, balancing for sex.

During sensitisation by students attending the University of Sierra Leone (USL) and Njala University (NU), all participants were provided with leak-proof specimen container, and instructions. The fresh stool samples were collected within a few hours, flooded with $10 \%$ formalin, shaken to arrest development of ova or larvae and an identification number for each sample was allocated. Samples were transported back and examined in laboratories at USL and NU. One slide per stool sample was prepared and examined by experienced parasitological examiners with the Kato-Katz thick smear technique 
using a $41.7 \mathrm{mg}$ template. Number of parasite eggs per slide was recorded and intensity of infection was calculated and expressed as eggs per gram (epg) of faeces. Individual infections with S. mansoni and STHs were classified as light, moderate or heavy according to the WHO classifications [27].

\section{Data analysis}

Results obtained were entered into computer using Epi info ${ }^{\circledR}$ software (version 3.5.1) and exported into SPSS (IBM, version 18) for analysis. The size of districts and the number of schools existing within each district varied significantly; therefore, sample weighting was applied during calculation. Sample weights were calculated for each district according to the ratio of the proportionally expected number of schools to be surveyed (total number of schools within a district $\times$ overall proportion of schools selected in seven districts) and the number of actually surveyed schools in each district. Prevalence and arithmetic mean intensity of infection among all children examined with 95\% confidence intervals (CIs) were obtained using the Complex Samples analysis in the SPSS, stratified by districts. The $95 \%$ CIs for prevalence were calculated using the Wilson score method without continuity correction after adjusting for sample weighting [28]. Differences in prevalence were compared using Chi-square test, and differences in intensity of infection were compared using Mann-Whitney test for two samples and Kruskal-Wallis test for multi groups. The coordinates of each sample site were documented using hand-held units of global positioning system. The prevalence maps of geographical distribution of each disease using observed point prevalence from each school were produced using ArcMap software (ESRI, version 9.3).

Ethical approval for the survey was obtained from the Ministry of Health and Sanitation, Sierra Leone and the Liverpool School of Tropical Medicine Research Ethics Committee. Community informed consent was obtained following discussion with District Medical Officers, Chiefdom school inspectors, head teachers and community-teachers associations. Verbal individual consent was obtained from parents/carers as literacy rates among parents are low in these locations.

\section{Results}

\section{Overall prevalence and intensity of infection}

A total of 1,760 faecal specimens were examined with two samples missing age and sex data, (males $51.4 \%$ and females $48.6 \%)$. The mean age ( \pm standard deviation) in male was $11.52 \pm 1.67$ and in female $11.30 \pm 1.68$.

Table 1 summarizes the overall prevalence and intensity of infection in the children examined. Overall $S$. mansoni prevalence was moderate: $40.2 \%$ (95\% CI: 37.9-
42.5\%) and also for hookworm: 31.2\% (95\% CI: 29.133.4\%). Overall prevalence for A. lumbricoides was low: 1.5\% (95\% CI: 1.1-2.2\%) and for T. trichiura: $2.5 \%$ (95\% CI: $1.9-3.4 \%$ ). There were $33.0 \%$ (95\% CI: $30.8-35.2 \%$ ) of children infected with any STHs, and 60.7\% (95\% CI: 58.4-63.0\%) of children infected with any of four parasites surveyed. There were no significant differences between sex in S. mansoni, A. lumbricoides or T. trichiura infection $(\mathrm{p}>0.05)$, but significantly more boys were infected with hookworms than girls $(\mathrm{p}=0.002)$. There was no difference between age groups in any of four infections $(\mathrm{p}>0.05)$.

Overall intensity of S. mansoni infection was 95.4 epg (95\% CI: 61.4-129.5 epg); hookworm: 53.0 epg (95\% CI: 38.4-67.7 epg); A. lumbricoides: 17.8 epg (95\% CI: 0.0-38.9 epg); and T. trichiura: $20.3 \mathrm{epg}$ (95\% CI: 0.0-48.5 epg). There was no significant difference in intensity of infection between sex or between ages in S. mansoni, A. lumbricoides or T. trichiura infection ( $\mathrm{p}>0.05$ ), but boys were more heavily infected with hookworms than girls $(\mathrm{p}<0.01)$. Based on the WHO classifications [27], there were $20.7 \%$ of children heavily or moderately infected with $S$. mansoni, while all infections with STHs were relatively light (details not shown).

\section{Geographical distribution}

Table 2 summarizes the prevalence and intensity of infection of $S$. mansoni and hookworm by district as these were the main parasites confirmed (data for $A$. lumbricoides and T. trichiura not shown due to low levels of infection). Figure 1 shows the point prevalence distribution of S. mansoni and STHs in the schools surveyed in the seven districts. S. mansoni infection was confirmed in all seven districts with high level of infection in the most north-easterly districts: Kono (median prevalence: 71.7\%; arithmetic mean intensity of infection: $250.3 \mathrm{epg})$, Kailahun (63.3\%; $120.2 \mathrm{epg})$, Kenema (46.7\%; 104.5 epg) and Koinadugu (41.9\%; $112.3 \mathrm{epg})$.

In Bo district where the 2008 mapping survey did not find $S$. mansoni infection it was confirmed in eight out of nine survey sites with prevalence ranging 3.3-63.3\% and a mean intensity of $17.8 \mathrm{epg}$ (95\% CI: 1.9-33.8). The focal distribution of $S$. mansoni was also confirmed in Tonkolili and Bombali with prevalence ranging from 3$90 \%$ and $0 \%-67.9 \%$ respectively and mean intensity of 32.1 epg (95\% CI: 1.6-62.6) and 80.5 epg (95\% CI: 9.2151.8) respectively.

Hookworm prevalence was high in Tonkolili (56.7\%; $139.9 \mathrm{epg}$ ) and Bo (50\%; $68.2 \mathrm{epg}$ ), moderate in Kailahun (16.7\%, 7.7 epg), Kenema (26.7\%, 38.8 epg), Kono (31.7\%, 29.9 epg) and Koinadugu (26.7\%, $25.7 \mathrm{epg}$ ) and low in Bombali (11.1\%, 12.5 epg). 
Table 1 Adjusted S. mansoni and STH prevalence and intensity of infection (95\% CI) in 9-14 years old in seven districts, Sierra Leone

\begin{tabular}{|c|c|c|c|c|c|c|}
\hline & & No of subjects & S. mansoni & Hookworm & A. lumbricoides & T. trichiura \\
\hline \multicolumn{7}{|l|}{ Prevalence (\%) } \\
\hline Overall prevalence & & 1760 & $40.2(37.9-42.5)$ & $31.2(29.1-33.4)$ & $1.5(1.1-2.2)$ & $2.5(1.9-3.4)$ \\
\hline \multicolumn{7}{|l|}{ Sex } \\
\hline & Boys & 904 & $41.7(38.5-45.0)$ & $35.3(32.3-38.5)$ & $1.3(0.8-2.3)$ & $2.2(1.4-3.4)$ \\
\hline & Girls & 854 & $38.5(35.3-41.8)$ & $27.0(24.1-30.1)$ & $1.8(1.1-2.9)$ & $2.9(2.0-4.3)$ \\
\hline \multicolumn{7}{|c|}{ Intensity of Infection (epg) } \\
\hline Overall mean epg & & 1760 & $95.4(61.4-129.5)$ & $53.0(38.4-67.7)$ & $17.8(0.0-38.9)$ & $20.3(0.0-48.5)$ \\
\hline \multicolumn{7}{|l|}{ Sex } \\
\hline & Boys & 904 & $96.6(64.2-129.0)$ & $60.2(43.9-76.5)$ & $11.8(1.0-22.7)$ & $12.2(0.0-27.5)$ \\
\hline & Girls & 854 & $94.2(56.0-132.4)$ & $45.7(24.3-67.2)$ & $24.1(0.0-66.5)$ & $28.9(0.0-83.8)$ \\
\hline
\end{tabular}

\section{Costs of the survey}

The field work was performed with the support of undergraduate students from the University of Sierra Leone and Njala University and laboratory work with support from Liverpool School of Tropical Medicine. The total expenditure on the survey, including travel, field work allowances, laboratory examinations etc was 18,375 US dollars, an equivalent of $\$ 311$ per site surveyed. This does not include the personnel salaries/wages.

\section{Discussion}

Planning and implementation of preventive chemotherapy strategy recommended by the WHO depends on a good understanding of endemic situation of the diseases in each district [29-31]. This is particularly the case for schistosomiasis because of its focal distribution in areas, close to snail-infected water sources which in Sierra Leone are altitude related [12]. The mapping survey conducted in 2008, prior to the MDA of the national integrated NTD control programme, gave an overall picture of S. mansoni and STH endemic situations in Sierra Leone [26]. Together with spatial prediction, the northeast half of the country was classified as highly endemic for S. mansoni, which required annual MDA for all school-age children plus adults at high risk. However, in the 2008 survey relatively few (four) sites per district were used, therefore there was a need to validate the predicted prevalence maps and confirm the PCT strategy in chiefdoms that were not surveyed. Furthermore, in three districts (Bo, Bombali and Tonkolili) it was unclear from the 2008 mapping whether MDA was justified and if so how frequently it should be performed.

Table 2 S. mansoni and hookworm median prevalence (inter-quantile range, minimum-maximum range) and arithmetic mean intensity of infection $(95 \% \mathrm{Cl})$ by districts, Sierra Leone

\begin{tabular}{|c|c|c|c|}
\hline & No of subjects & S. mansoni & Hookworm \\
\hline \multicolumn{4}{|l|}{ Prevalence (\%) } \\
\hline Bo & 269 & $6.7(3.3-13.3,0.0-63.3)$ & $50.0(17.2-53.3,6.7-66.7)$ \\
\hline Bombali & 261 & $13.3(3.3-29.6,0.0-67.9)$ & $11.1(10.0-17.9,3.4-46.7)$ \\
\hline Kailahun & 270 & $63.3(43.3-66.7,43.3-73.3)$ & $16.7(10.0-20.0,3.3-23.3)$ \\
\hline Kenema & 269 & $46.7(23.3-50.0,17.2-96.7)$ & $26.7(16.7-36.7,0.0-60.0)$ \\
\hline Koinadugu & 211 & $41.9(30.0-83.3,13.3-93.3)$ & $26.7(16.7-35.0,9.7-46.7)$ \\
\hline Kono & 240 & 71.7 (58.3-82.5, 53.3-93.3) & $31.7(22.5-36.7,10.0-40.0)$ \\
\hline Tonkolili & 240 & $18.3(14.2-74.2,3.3-90.0)$ & $56.7(50.0-57.5,26.7-66.7)$ \\
\hline \multicolumn{4}{|l|}{ Intensity of Infection (epg) } \\
\hline Bo & 269 & $17.8(1.9-33.8)$ & $68.2(36.5-99.9)$ \\
\hline Bombali & 261 & $32.1(1.6-62.6)$ & $12.5(0.0-28.6)$ \\
\hline Kailahun & 270 & $120.2(31.5-208.8)$ & $7.7(2.8-12.6)$ \\
\hline Kenema & 269 & $104.5(0.0-210.8)$ & $38.8(13.3-64.3)$ \\
\hline Koinadugu & 211 & $112.3(26.7-197.9)$ & $25.7(15.3-36.1)$ \\
\hline Kono & 240 & $250.3(95.0-405.6)$ & $29.9(17.5-42.3)$ \\
\hline Tonkolili & 240 & $80.5(9.2-151.8)$ & $139.9(72.0-207.8)$ \\
\hline
\end{tabular}




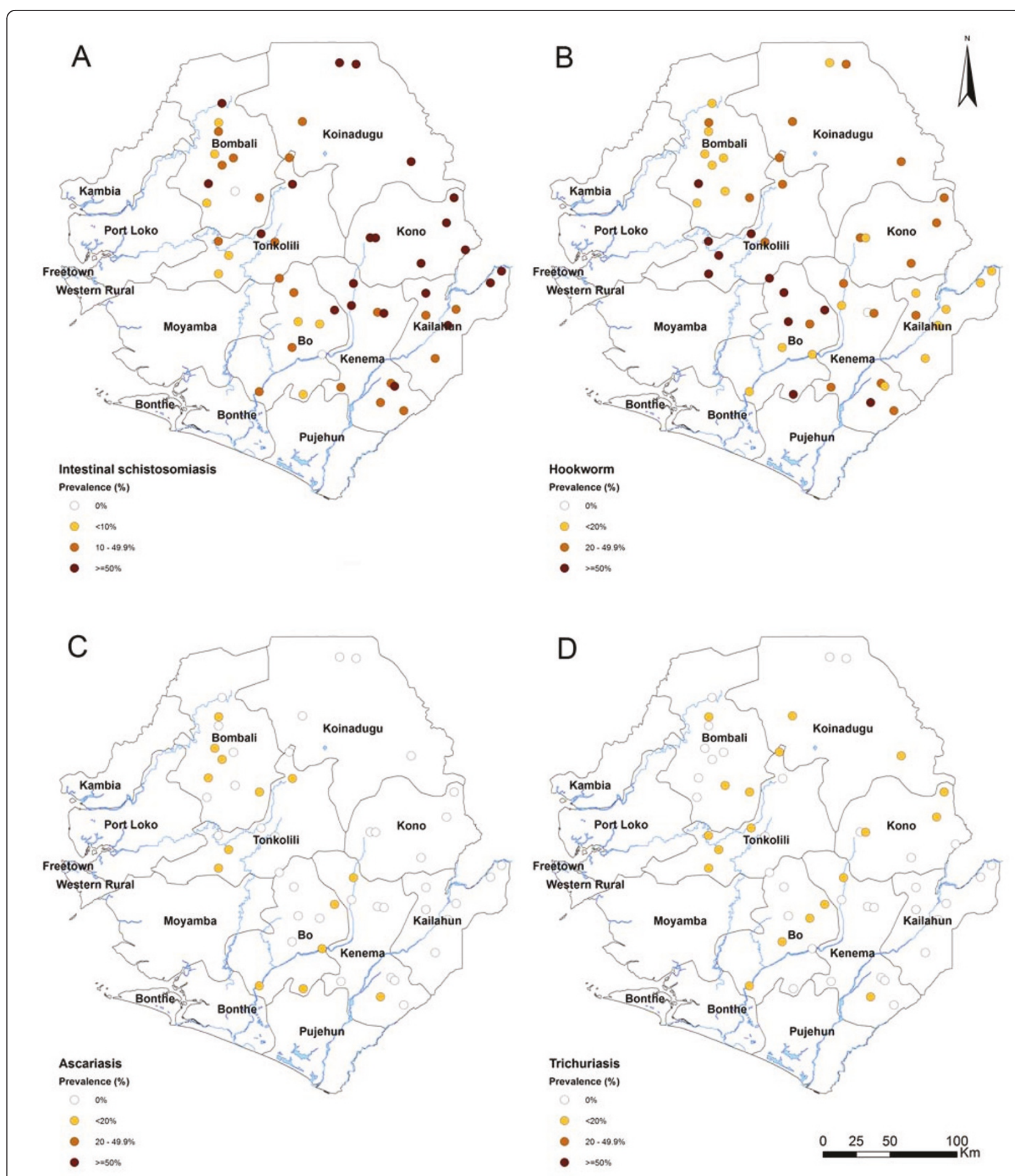

Figure 1 Geographical distribution of intestinal schistosomiasis and soil-transmitted helminthiasis in selected schools in seven districts, Sierra Leone. Observed point prevalence is shown by locations of the selected schools: (A) S. mansoni, (B) Hookworm, (C) A. lumbricoides, and (D) T. trichiura. 
The results of this current survey confirmed the high endemicity of S. mansoni in Kailahun, Kenema, Koinadugu and Kono districts and in Eastern chiefdoms of Bombali and Tonkolili districts, suggesting a convincing prediction by the spatial analysis in these areas. The results also confirmed that some chiefdoms in Bo district were indeed endemic and therefore qualified for MDA. This highlights the importance of sampling methods in schistosomiasis survey to meet the nature of focal transmission of the disease.

The results also provided valuable data on the intensity of infection of S. mansoni and STHs in school aged children in these areas which were not recorded in the 2008 mapping survey. School aged children in Kailahun, Kenema, Koinadugu and Kono districts are relatively heavily infected with $S$. mansoni with arithmetic mean intensities of infection of over 100 epg. There are $20.7 \%$ of children heavily or moderately infected with $S$. mansoni in these seven districts. This highlights the need for intervention to prevent severe morbidity in their later life [32]. Based on these survey data, MDA was planned and these highly endemic districts have so far received two rounds of treatment In 2009 school-going children were targeted, and 562,980 received praziquantel and 549,701 received mebendazole. In 2010, all school-aged children and at risk adults were targeted, and 1,826,284 received praziquantel and 1,000,042 received albendazole [33].

The focal distribution of S. mansoni is most clearly demonstrated in Bo, Bombali and Tonkolili with prevalence widely ranging from chiefdom to chiefdom. In the context of such districts in Sierra Leone, it is important to plan the implementation strategy according to the chiefdoms rather than the whole districts. This complementary survey provided such a tool to enable the national program managers and district health teams to plan and refine their MDA activities in conjunction with the 2008 mapping results. This is a clear demonstration that a detailed mapping survey with sufficient number of survey sites across the endemic areas, in particular for focal diseases such as schistosomiasis, is needed in order to better plan the control activities. There is a dilemma currently for the national teams in terms of the need of detailed mapping and the funds available. The current survey of about $\$ 310$ per site also demonstrated the affordability of such mapping surveys with the contributions from all parties involved and national resources mobilized.

Low prevalence and intensity of A. lumbricoides and T. trichiura and moderate prevalence and light intensity with hookworm highlight remarkable reductions in comparison with pre-war studies [18-20,23,24]. The impact on STH of the annual distribution of ivermectin and albendazole in the NTDCP's lymphatic filariasis elimination programme since 2007 and various schoolbased deworming programs performed by the Wood Food Program and NGOs since 2004 has been discussed previously [26,33]. Although of light intensity, the moderate prevalence of hookworm infections and widespread anaemia in children in Sierra Leone according to the national data confirm the desirability of using albendazole as the drug of choice as albendazole has a better cure rate for hookworm infections [34].

The current survey provided a significantly improved tool for planning the MDA activities in each district, however there are certain limitations in the methodology used. Firstly, the sampling method of survey sites based upon accessibility, though not uncommon for schistosomiasis, may not represent the true endemicity level in chiefdoms, as those schools in hard-to-reach locations may be heavily affected by schistosomiasis. Secondly, by adding formalin to the stool samples, it may have diluted the stool samples and caused underestimation of the results. However, considering the rural conditions in the post-war Sierra Leone, it was not possible to process and examine the stool samples on site, and therefore, preservation of the samples was a necessary trade-off to avoid disintegration of helminth, particularly hookworm, eggs. Thirdly, only one Kato-Katz slide was used for the diagnosis, and the low sensitivity of this method may have caused underestimation of the prevalence and slight overestimation of the intensity of infection. However, considering the morbidity control strategy for schistosomiasis and STH by preventive chemotherapy in the current national control programme, such slight misestimation would not make much difference to the overall objectives of the national programme.

\section{Conclusions}

The current complementary survey confirmed that $S$. mansoni is indeed endemic across the northeast half of Sierra Leone, particularly in Kailahun, Kenema, Koinadugu, Kono, North-eastern Tonkolili and Eastern Bombali. It also confirmed that S. mansoni is prevalent in Bo district as the local knowledge anticipated, highlighting the importance of sampling survey sites for schistosomiasis survey according to the local knowledge and environmental situations. There are over $20 \%$ of children heavily or moderately infected with $S$. mansoni in these seven districts, justifying the need for MDA to prevent severe morbidity in their later life. This study clarified that MDA was justified annually throughout Kailahun, Kenema, Koinadugu, Kono, North-eastern Tonkolili, Eastern Bombali and in certain chiefdoms in Bo. The survey demonstrated that sufficient number of survey sites for schistosomiasis mapping in each district should be used to provide a better national planning of MDA activities. 


\section{Acknowledgements}

This study was made possible with funding from the United States Agency for International Development (USAID). USAID support for NTD control in Sierra Leone is provided through a grant to Helen Keller International as part of the Neglected Tropical Disease (NTD) Control Program led by RTI International under Cooperative Agreement No. GHS-A-00-06-00006-00. The contents are the responsibility of the authors and do not necessarily reflect the views of USAID or the United States Government. The authors would also like to thank Hamid Turay, Professor Karim and Mrs Kaiwa, University of Sierra Leone, and Professor Roberts and Mr Abu Conteh, Njala University for collaboration during the field and laboratory work.

\section{Author details}

${ }^{1}$ Helen Keller International, Freetown, Sierra Leone. 'Liverpool School of Tropical Medicine, Liverpool, UK. ${ }^{3}$ Faculty of Pure and Applied Science, University of Sierra Leone. ${ }^{4}$ School of Community Health Sciences, Njala University, Sierra Leone. ${ }^{5}$ Helen Keller International, Regional Office for Africa, Dakar, Senegal.

\section{Authors' contributions}

$\mathrm{MH}$ conceived, designed, supervised the study and revised the paper; JP, EN and MS coordinated the field data collection; ND, AW, JP performed the laboratory investigations, ND and AW conducted data entry and initial data analysis; YZ conducted final data analysis using SPSS and ArcMap, and MH and $Y Z$ drafted and revised the paper. GB, MB supervised ND and AW and reviewed the paper. All authors reviewed and approved the final manuscript.

\section{Competing interests}

The authors declare that they have no competing interests.

Received: 31 March 2011 Accepted: 6 June 2011 Published: 6 June 2011

\section{References}

1. Bethony J, Brooker S, Albonico M, Geiger SM, Loukas A, Diemert D, Hotez PJ: Soil-transmitted helminth infections: ascariasis, trichuriasis, and hookworm. Lancet 2006, 367(9521):1521-1532

2. Steinmann P, Keiser J, Bos R, Tanner M, Utzinger J: Schistosomiasis and water resources development: systematic review, meta-analysis, and estimates of people at risk. Lancet Infect Dis 2006, 6(7):411-425.

3. Hotez PJ, Molyneux DH, Fenwick A, Kumaresan J, Sachs SE, Sachs JD, Savioli L: Control of neglected tropical diseases. N Engl J Med 2007, 357(10):1018-1027.

4. Hotez P, Gupta R, Mahoney R, Poste G: Incorporating appropriate technology into North American schools of public health. Rev Panam Salud Publica 2006, 19(2):118-123.

5. Hotez PJ, Brindley PJ, Bethony JM, King CH, Pearce EJ, Jacobson J: Helminth infections: the great neglected tropical diseases. $J$ Clin Invest 2008, 118(4):1311-1321

6. King $\mathrm{CH}$, Dickman $\mathrm{K}$, Tisch DJ: Reassessment of the cost of chronic helmintic infection: a meta-analysis of disability-related outcomes in endemic schistosomiasis. Lancet 2005, 365(9470):1561-1569.

7. Savioli L, Albonico M, Engels D, Montresor A: Progress in the prevention and control of schistosomiasis and soil-transmitted helminthiasis. Parasitol Int 2004, 53(2):103-113.

8. Crompton DW, Nesheim MC: Nutritional impact of intestinal helminthiasis during the human life cycle. Annu Rev Nutr 2002, 22:35-59.

9. Guyatt H: Do intestinal nematodes affect productivity in adulthood? Parasitol Today 2000, 16(4):153-158.

10. Brooker S, Clements AC, Bundy DA: Global epidemiology, ecology and control of soil-transmitted helminth infections. Adv Parasitol 2006 62:221-261.

11. Zhang Y, MacArthur C, Mubila L, Baker S: Control of neglected tropical diseases needs a long-term commitment. BMC Med 2010, 8:67.

12. WHO: Atlas of Global distribution of schistosomiasis Geneva: World Health Organisation; 1987

13. Onabamiro SD: Studies on schistosomiasis in Sierra Leone. I. Ann Trop Med Parasitol 1971, 65(4):497-504.

14. Onabamiro SD: Studies in schistosomiasis in Sierra Leone. II. Seasonal fluctuation in the population density of Bulinus (Physopsis) globosus and Bulinus forskalii in a schistosomiasis endemic town in Sierra Leone. Ann Trop Med Parasitol 1972, 66(3):375-383.
15. Porter JC, Whitworth JA: Onchocerca volvulus and Schistosoma haematobium infection in southern Sierra Leone. Lancet 1994, 344(8936):1581-1582

16. White PT, Gbakima AA, Amara SV: Schistosoma mansoni in Sierra Leone: an invader extending its range? Ann Trop Med Parasitol 1989, 83(2):191-193.

17. Gbakima AA, Konteh R, Kallon M, Mansaray H, Sahr F, Bah ZJ, Spencer A Luckay A: Intestinal protozoa and intestinal helminthic infections in displacement camps in Sierra Leone. Afr J Med Med Sci 2007, 36(1):1-9.

18. Gbakima AA, Sahr F: Intestinal parasitic infections among rural farming communities in eastern Sierra Leone. Afr J Med Med Sci 1995 24(2):195-200

19. Gbakima AA, Sherpard M, White PT: Intestinal helminth infections in rural school children in Njala, Sierra Leone. East Afr Med J 1994, 71(12):792-796

20. Gbakima AA: Intestinal parasitic infections and swamp development in Sierra Leone. Afr J Health Sci 1994, 1(4):175-178.

21. Gbakima AA: Inland valley swamp rice development: malaria, schistosomiasis, onchocerciasis in south central Sierra Leone. Public Health 1994, 108(2):149-157.

22. Williams RA, Koroma MM, Hodges M: Comparison of albendazole and levamisole chemotherapy on prevalence and intensity of common soiltransmitted helminth infections in school children, Sierra Leone. West Afr J Med 1997, 16(3):179-183.

23. Koroma MM, Williams RA, de la Haye RR, Hodges M: Effects of albendazole on growth of primary school children and the prevalence and intensity of soil-transmitted helminths in Sierra Leone. J Trop Pediatr 1996, 42(6):371-372.

24. Bayoh MN, Hodges M: Soil-transmitted helminth infections in three isolated communities in Sierra Leone. J Sierra Leone Med Dental Association 1994, 8:1-7.

25. Bayoh MN, Hodges M: Effect of levamisole chemotherapy on geohelminthiases in Sierra Leone. J Sierra Leone Med Dental Association 1993, 6:1-4.

26. Koroma JB, Peterson J, Gbakima AA, Nylander FE, Sahr F, Soares Magalhaes RJ, Zhang Y, Hodges MH: Geographical distribution of intestinal schistosomiasis and soil-transmitted helminthiasis and preventive chemotherapy strategies in sierra leone. PLoS Negl Trop Dis 2010, 4(11):e891.

27. WHO: Prevention and control of schistosomiasis and soil-transmitted helminthiasis. WHO Technical Report Series 2002, 912.

28. Newcombe RG: Two-sided confidence intervals for the single proportion: comparison of seven methods. Stat Med 1998, 17(8):857-872.

29. WHO: Preventive Chemotherapy in human helminthiasis: coordinated use of anthelminthic drugs in control interventions Geneva: World Health Organization; 2006

30. Kapito-Tembo AP, Mwapasa V, Meshnick SR, Samanyika Y, Banda D, Bowie C, Radke S: Prevalence distribution and risk factors for Schistosoma hematobium infection among school children in Blantyre, Malawi. PLOS Negl Trop Dis 2009, 3(1):e361.

31. Clements AC, Lwambo NJ, Blair L, Nyandindi U, Kaatano G, Kinung'hi S, Webster JP, Fenwick A, Brooker S: Bayesian spatial analysis and disease mapping: tools to enhance planning and implementation of a schistosomiasis control programme in Tanzania. Trop Med Int Health 2006, 11(4):490-503.

32. Fenwick A, Zhang Y: Schistosomiasis. In Infectious Diseases.. 3 edition. Edited by: Cohen J, Opal SM. Powderly WG: Elsevier; 2010:

33. Hodges M, Koroma JB, Sonnie M, Kennedy N, Cotter E, MacArthur C: Neglected Tropical Disease Control in Post-war Sierra Leone. International Health 2011.

34. Keiser J, Utzinger J: Efficacy of current drugs against soil-transmitted helminth infections: systematic review and meta-analysis. JAMA 2008, 299(16):1937-1948

doi:10.1186/1756-3305-4-97

Cite this article as: Hodges et al:: Improved mapping strategy to better inform policy on the control of schistosomiasis and soil-transmitted helminthiasis in Sierra Leone. Parasites \& Vectors 2011 4:97. 